\title{
THE (3 + 1)-DIMENSIONAL FRACTIONAL MODIFIED KdV-ZAKHAROV-KUZNETSOV EQUATION BY USING THE IMPROVED GENERALIZED TANH-COTH METHOD
}

\section{MONTRI TORVATTANABUN, CHANTANA SIMTRAKANKUL and SOMJATE DUANGPITHAK}

Department of Mathematics

Faculty of Science and Technology

Loei Rajabhat University

Loei 42000

Thailand

e-mail: montri_kai@windowslive.com

\begin{abstract}
In this paper, the improved generalized tanh-coth method is used to establish exact travelling wave solutions of the $(3+1)$-dimensional fractional modified KdV-Zakharov-Kuznetsov equation. The fractional derivative version of Yang modified, linked with fractional complex transform is employed to reduce fractional differential equations into the corresponding ordinary differential equations. The results confirm that proposed method is reliability and effectiveness of the current method.
\end{abstract}

2010 Mathematics Subject Classification: 35E05, 35D99, 35A20, 35Q51.

Keywords and phrases: the improved generalized tanh-coth method, exact travelling wave solutions, the (3+1)-dimensional fractional modified KdV-Zakharov-Kuznetsov equation.

Received March 22, 2019

(ㄷ) 2019 Scientific Advances Publishers 


\section{Introduction}

Fractional differential equations (FDEs) arise in numerous problems of science, control theory, engineering, biology, physics, mathematics, chemistry and other areas. Therefore, exact solution methods of the fractional differential equations have become more important. Many researchers have used diverse methods to get exact solutions, such as, the functional variable method, Lie symmetries, Riccati-sub equation method, first integral method, improved fractional sub-equation method, the exp-function method and so on [1-6].

Another powerful method has been presented by Maliet [7], who had customized the tanh technique and called the tanh method. In 2002, Fan and Hona [8] extended the tanh method which is called the extended tanh method. In 2006, Wazwaz [9] extended and improved this method which is called the tanh-coth method. In 2008, Gomez and Salas [10] improved and generalized this method which is called the improved generalized tanh-coth method. After wards, several researchers applied this method to obtain exact solutions for nonlinear PDEs [10-13].

The $(3+1)$-dimensional fractional modified KdV-Zakharov-Kuznetsov equation can be derived for the behaviour of weakly nonlinear ionacoustic waves with components of two negative ions of different temperatures. It is the aim of this work, we use the improved generalized tanh-coth method to obtain exact travelling wave solutions of the $(3+1)$-dimensional fractional modified KdV-Zakharov-Kuznetsov equation.

The remainder of this paper is organized as follows. The formal definitions and properties of local fractional derivative have been explained in Section 2 and we briefly describe the extended and improved this method in Section 3. In Section 4, the improved generalized tanhcoth method is applied to the $(3+1)$-dimensional fractional modified KdV-Zakharov-Kuznetsov equation. Some conclusions are given in the last section. 


\section{Local Fractional Derivative and its Properties}

The summary of local fractional derivative of order which is used further in this paper is defined by the following expression [14, 15]:

$$
D^{\alpha} f\left(t_{0}\right)=\frac{d^{\alpha} f(t)}{d t^{\alpha}}=\lim _{t \rightarrow t_{0}} \frac{\Delta^{\alpha}\left(f(t)-f\left(t_{0}\right)\right)}{\left(t-t_{0}\right)^{\alpha}}, \quad 0<\alpha \leq 1
$$

in which $\Delta^{\alpha}\left(f(t)-f\left(t_{0}\right)\right)$ is the $\Delta \alpha$ function defined by

$$
\Delta^{\alpha}\left(f(t)-f\left(t_{0}\right)\right) \cong \gamma(1+\alpha)\left(f(t)-f\left(t_{0}\right)\right)
$$

Some important properties of the local derivative famous formula can be listed as follows:

$$
\begin{aligned}
D^{\alpha}\left(f_{1}(t) \pm f_{2}(t)\right) & =D^{\alpha}\left(f_{1}(t)\right) \pm D^{\alpha}\left(f_{2}(t)\right), \\
D^{\alpha}(c f(t)) & =c D^{\alpha}(f(t)), \quad c=\text { constant, } \\
D^{\alpha} t^{\beta} & =\frac{\Gamma(1+\beta)}{\Gamma(1+\beta-\alpha)} t^{\beta-\alpha}, \quad \beta \geq \alpha>0, \\
D^{\alpha}(f(g(t))) & =D^{\alpha}(g(t)) g^{(1)}(t) .
\end{aligned}
$$

\section{The Improved Generalized Tanh-Coth Method}

We suppose that given nonlinear partial differential equation for $u(x, y, z, t)$ to be in from

$$
F\left(u, u_{x}, u_{t}, u_{y}, u_{z}, \ldots\right)=0
$$

which can be converted to an ODE.

$$
O\left(u, u^{\prime}, u^{\prime \prime}, u^{\prime \prime \prime}, \ldots\right)=0,
$$


by the travelling wave transformation is given by $u=u(\xi), \xi=a x+b y+$ $c z-v t$. We seek the exact solution of Equation (6) that can be expressed in the following form:

$$
u(\xi)=\sum_{n=0}^{M} A_{n} \phi(\xi)^{n}+\sum_{n=M+1}^{2 M} A_{n} \phi(\xi)^{M-n},
$$

where $M$ is a positive integer that will be determined by balancing the highest order derivative term with the highest order nonlinear term. The coefficients $A_{n}$ are constants $\left(A_{M} \neq 0\right.$ and $\left.A_{-M} \neq 0\right)$ that are determined later while the new variable $\phi(\xi)$ is the solution to the generalized Riccati equation

$$
\phi^{\prime}(\xi)=g_{0}+g_{1} \phi(\xi)+g_{2}(\phi(\xi))^{2},
$$

where $g_{0}, g_{1}$, and $g_{2}$ are constants. The solutions of generalized Riccati equation are given by [10].

Case 1 (Exponential function solution): When $g_{0}=0$,

$$
\phi(\xi)=\frac{g_{1}}{-g_{2}+g_{1} e^{-g_{1} \xi}} .
$$

Case 2 (Trigonometric and hyperbolic function solutions): When $g_{1}=0$,

$$
\phi(\xi)=\left\{\begin{array}{cc}
\frac{\sqrt{g_{0} g_{2}}}{g_{2}} \tan \left(\sqrt{g_{0} g_{2}} \xi\right), & g_{0}>0, g_{2}>0, \\
\frac{\sqrt{g_{0} g_{2}}}{g_{2}} \tanh \left(\sqrt{g_{0} g_{2}} \xi\right), & g_{0}>0, g_{2}<0, \\
\frac{\sqrt{-g_{0} g_{2}}}{g_{2}} \tanh \left(-\sqrt{-g_{0} g_{2}} \xi\right), & g_{0}<0, g_{2}>0, \\
\frac{\sqrt{g_{0} g_{2}}}{g_{2}} \tan \left(-\sqrt{g_{0} g_{2}} \xi\right), & g_{0}<0, g_{2}<0 .
\end{array}\right.
$$


Case 3 (Exponential function solution): When $g_{2}=0$,

$$
\phi(\xi)=\frac{-g_{0}+g_{1} e^{g_{1} \xi}}{g_{1}} .
$$

Case 4 (Rational function solution): When $g_{0}=0, g_{1}=0$,

$$
\phi(\xi)=-\frac{1}{g_{2} \xi}
$$

Case 5 (Rational function solution): When $g_{1}^{2} \neq 0$ and $g_{1}^{2}=4 g_{2} g_{0}$,

$$
\phi(\xi)=-\frac{2 g_{0}\left(g_{1} \xi+2\right)}{g_{1}^{2} \xi} .
$$

Case 6 (Trigonometric function solution): When $g_{1}^{2}<4 g_{0} g_{2}$ and $g_{2} \neq 0$,

$$
\phi(\xi)=\frac{\sqrt{4 g_{0} g_{2}-g_{1}^{2}} \tan \left(\frac{1}{2} \sqrt{4 g_{0} g_{2}-g_{1}^{2}} \xi\right)-g_{1}}{2 g_{2}} .
$$

Case 7 (Hyperbolic function solution): When $g_{1}^{2}>4 g_{0} g_{2}$ and $g_{2} \neq 0$,

$$
\phi(\xi)=\frac{\sqrt{g_{1}^{2}-4 g_{0} g_{2}} \tanh \left(\frac{1}{2} \sqrt{g_{1}^{2}-4 g_{0} g_{2}} \xi\right)-g_{1}}{2 g_{2}} .
$$

We then substitute Equation (7) into Equation (6) and collect all terms with the same order of $\phi^{j}(\xi)$ together. We can get a polynomial in $\phi(\xi)$. Equating each coefficient of the polynomial to zero yields a system of algebraic equations involving the parameters $A_{i}, g_{0}, g_{1}$, and $g_{2}$. Solving the equation system, we can construct a variety of exact solutions of Equation (5). 


\section{Application of the Improved Generalized \\ Tanh-Coth Method}

Consider the $(3+1)$-dimensional fractional modified KdV-ZakharovKuznetsov equation ([2-6], [16])

$$
D_{t}^{\alpha} u+\delta u^{2} D_{x}^{\alpha} u+D_{x}^{3 \alpha} u+D_{x}^{\alpha} D_{y}^{2 \alpha} u+D_{x}^{\alpha} D_{z}^{2 \alpha} u=0,
$$

where $0<\alpha \leq 1$. We use the wave transformation $u(x, y, z, t)=U(\xi)$, $\xi=\frac{a x^{\alpha}}{\Gamma(1+\alpha)}+\frac{b y^{\alpha}}{\Gamma(1+\alpha)}+\frac{c z^{\alpha}}{\Gamma(1+\alpha)}-\frac{v t^{\alpha}}{\Gamma(1+\alpha)}$ to reduce (16) to following ODE:

$$
-v U^{\prime}+\delta a U^{2} U^{\prime}+\left(a^{3}+a b^{2}+a c^{2}\right) U^{\prime \prime \prime}=0
$$

Integrating the resultant once, we obtain

$$
-v U+\frac{\delta a}{3} U^{3}+\left(a^{3}+a b^{2}+a c^{2}\right) U^{\prime \prime}=0 .
$$

Balancing the highest order term $U^{\prime \prime}$ with the highest order nonlinear term $U^{3}$ in (13), we have $M+2=3 M$, then $M=1$. Consequently, we set

$$
U(\xi)=A_{0}+A_{1} \phi(\xi)+A_{2} \phi(\xi)^{-1} .
$$

Substituting Equation (19) into Equation (18) and equating all the coefficients of power of $\phi(\xi)$ to be zero, we obtain a system of algebraic equations involving the parameters $A_{0}, A_{1}, A_{2}$, and $v$.

$$
\begin{gathered}
\phi(\xi)^{-3}: 2 a^{3} A_{2} \alpha^{2}+\frac{1}{3} \delta a A_{2}{ }^{3}+2 b^{2} a A_{2} \alpha^{2}+2 c^{2} a A_{2} \alpha^{2}=0, \\
\phi(\xi)^{-2}: 3 a^{3} A_{2} \alpha \beta+3 b^{2} a A_{2} \alpha \beta+3 c^{2} a A_{2} \alpha \beta+\delta a A_{2}{ }^{2} A_{0}=0, \\
\phi(\xi)^{-1}: 2 A_{2} a^{3} \alpha \gamma+A_{2} a^{3} \beta^{2}+2 A_{2} a b^{2} \alpha \gamma+A_{2} a b^{2} \beta^{2}+2 A_{2} a c^{2} \alpha \gamma \\
+A_{2} a c^{2} \beta^{2}+{A_{0}}^{2} A_{2} a \delta+A_{1} A_{2}{ }^{2} a \delta-A_{2} v=0,
\end{gathered}
$$




$$
\begin{gathered}
\phi(\xi)^{0}: a^{3} A_{1} \beta \alpha+b^{2} a A_{1} \beta \alpha+c^{2} a A_{1} \beta \alpha+\frac{1}{3} \delta a A_{0}^{3}+b^{2} a A_{2} \beta \gamma \\
+c^{2} a A_{2} \beta \gamma-v A_{0}+a^{3} A_{2} \beta \gamma+2 \delta a A_{2} A_{0} A_{1}=0, \\
\phi(\xi)^{1}: 2 A_{1} a^{3} \alpha \gamma+A_{1} a^{3} \beta^{2}+2 A_{1} a b^{2} \alpha \gamma+A_{1} a b^{2} \beta^{2}+2 A_{1} a c^{2} \alpha \gamma \\
+A_{1} a c^{2} \beta^{2}+A_{0}^{2} A_{1} a \delta+A_{1}^{2} A_{2} a \delta-A_{1} v=0, \\
\phi(\xi)^{2}: 3 A_{1} a^{3} \beta \gamma+3 A_{1} a b^{2} \beta \gamma+3 A_{1} a c^{2} \beta \gamma+A_{0} A_{1}^{2} a \delta=0, \\
\phi(\xi)^{3}: 2 a^{3} A_{1} g_{2}^{2}+\frac{1}{3} \delta a A_{1}^{3}+2 c^{2} a A_{1} \gamma^{2}+2 b^{2} a A_{1} \gamma^{2}=0 .
\end{gathered}
$$

We solve the system of algebraic equations with the aid of Maple, using Equations (20)-(26), that obtain following:

First set

$$
\begin{aligned}
& A_{0}= \pm \frac{1}{2} \sqrt{-\frac{6 a^{2}+6 b^{2}+6 c^{2}}{\delta}} \beta, A_{1}=0, \\
& A_{2}= \pm \alpha \sqrt{-\frac{6 a^{2}+6 b^{2}+6 c^{2}}{\delta}}, v=\frac{a}{2}\left(a^{2}+b^{2}+c^{2}\right)\left(4 g_{2} \alpha-\beta^{2}\right) .
\end{aligned}
$$

Case 1 (Trigonometric and hyperbolic function solutions): When $\beta=0$,

$$
\begin{aligned}
u_{1,2}= & \pm \frac{\gamma \alpha}{\sqrt{\gamma \alpha}} \sqrt{-\frac{6 a^{2}+6 b^{2}+6 c^{2}}{\delta}} \\
& \times \cot \left(\sqrt { \gamma \alpha } \left(\frac{a x^{\alpha}}{\Gamma(1+\alpha)}+\frac{b y^{\alpha}}{\Gamma(1+\alpha)}+\frac{c z^{\alpha}}{\Gamma(1+\alpha)}\right.\right. \\
& \left.\left.-\left(a^{2} \gamma+b^{2} g_{2}+c^{2} \gamma\right) \frac{2 \alpha a t^{\alpha}}{\Gamma(1+\alpha)}\right)\right),
\end{aligned}
$$




$$
\begin{aligned}
u_{3,4}= & \pm \frac{\gamma \alpha}{\sqrt{-\gamma \alpha}} \sqrt{-\frac{6 a^{2}+6 b^{2}+6 c^{2}}{\delta}} \\
& \times \operatorname{coth}\left(\sqrt { \gamma \alpha } \left(\frac{a x^{\alpha}}{\Gamma(1+\alpha)}+\frac{b y^{\alpha}}{\Gamma(1+\alpha)}+\frac{c z^{\alpha}}{\Gamma(1+\alpha)}\right.\right. \\
& \left.\left.-\left(a^{2} \gamma+b^{2} g_{2}+c^{2} \gamma\right) \frac{2 \alpha a t^{\alpha}}{\Gamma(1+\alpha)}\right)\right) .
\end{aligned}
$$

Case 2 (Exponential function solution): When $\gamma=0$,

$$
\begin{aligned}
u_{5,6}= & \pm \sqrt{-\frac{6\left(a^{2}+b^{2}+c^{2}\right)}{\delta}} \beta \\
& \times\left(\frac{\alpha}{\left.-\alpha+\beta e^{\beta\left(\frac{a x^{\alpha}}{\Gamma(1+\alpha)}+\frac{b y^{\alpha}}{\Gamma(1+\alpha)}+\frac{c z^{\alpha}}{\Gamma(1+\alpha)}+\frac{1}{2}\left(a^{2} \beta^{2}+b^{2} \beta^{2}+c^{2} \beta^{2}\right) \frac{a t^{\alpha}}{\Gamma(1+\alpha)}\right)}+\frac{1}{2}\right) .} .\right.
\end{aligned}
$$

Case 3 (Trigonometric function solution): When $\beta^{2}<4 \alpha \gamma$ and $\gamma \neq 0$,

$$
\begin{aligned}
& u_{7,8}= \pm \sqrt{-\frac{6 a^{2}+6 b^{2}+6 c^{2}}{\delta\left(4 \gamma \alpha-\beta^{2}\right)}} \\
& \left(\frac{2 \gamma \alpha}{\tan \left(\frac { 1 } { 2 } \sqrt { 4 g _ { 2 } \alpha - \beta ^ { 2 } } \left(\frac{a x^{\alpha}}{\Gamma(1+\alpha)}+\frac{b y^{\alpha}}{\Gamma(1+\alpha)}+\frac{c z^{\alpha}}{\Gamma(1+\alpha)}\right.\right.}\right. \\
& \left.\frac{\left.\left.-\frac{1}{2}\left(a^{2}+b^{2}+c^{2}\right)\left(4 \gamma \alpha-\beta^{2}\right) \frac{a t^{\alpha}}{\Gamma(1+\alpha)}\right)\right)-\frac{\beta}{\sqrt{4 \gamma \alpha-\beta^{2}}}}{2}+\frac{\sqrt{4 \gamma \alpha-\beta^{2}} \beta}{2}\right)
\end{aligned}
$$


Case 4 (Hyperbolic function solution): When $\beta^{2}>4 \alpha \gamma$ and $\gamma \neq 0$,

$$
\begin{gathered}
u_{9,10}= \pm \sqrt{-\frac{6 a^{2}+6 b^{2}+6 c^{2}}{\delta\left(-4 \gamma \alpha+\beta^{2}\right)}} \\
\left(\frac{2 \gamma \alpha}{\tanh \left(\frac { 1 } { 2 } \sqrt { - 4 \gamma \alpha + \beta ^ { 2 } } \left(\frac{a x^{\alpha}}{\Gamma(1+\alpha)}+\frac{b y^{\alpha}}{\Gamma(1+\alpha)}+\frac{c z^{\alpha}}{\Gamma(1+\alpha)}\right.\right.}\right. \\
\left.\frac{\left.\left.-\frac{1}{2}\left(a^{2}+b^{2}+c^{2}\right)\left(4 \gamma \alpha-\beta^{2}\right) \frac{a t^{\alpha}}{\Gamma(1+\alpha)}\right)\right)-\frac{\beta}{\sqrt{-4 \gamma \alpha+\beta^{2}}}}{2}-\frac{\sqrt{4 \gamma \alpha+\beta^{2}} \beta}{2}\right) .
\end{gathered}
$$

Second set

$$
\begin{gathered}
A_{0}= \pm \frac{1}{2} \sqrt{-\frac{6 a^{2}+6 b^{2}+6 c^{2}}{\delta}} \beta, A_{1}= \pm \sqrt{-\frac{6 a^{2}+6 b^{2}+6 c^{2}}{\delta}} \gamma \\
A_{2}=0, v=\frac{a}{2}\left(a^{2}+b^{2}+c^{2}\right)\left(4 \gamma \alpha-\beta^{2}\right) .
\end{gathered}
$$

Case 1 (Exponential function solution): When $\alpha=0$,

$$
\begin{aligned}
u_{11,12}= & \pm \sqrt{-\frac{6 a^{2}+6 b^{2}+6 c^{2}}{\delta}} \beta \\
& \times\left(\begin{array}{c}
\left.\frac{1}{2}+\frac{\gamma}{-\gamma+\beta e^{-\beta\left(\frac{a x^{\alpha}}{\Gamma(1+\alpha)}+\frac{b y^{\alpha}}{\Gamma(1+\alpha)}+\frac{c z^{\alpha}}{\Gamma(1+\alpha)}+\frac{1}{2}\left(a^{2} \beta^{2}+b^{2} \beta^{2}+c^{2} \beta^{2}\right) \frac{a t^{\alpha}}{\Gamma(1+\alpha)}\right.}}\right)
\end{array} .\right.
\end{aligned}
$$


Case 2 (Trigonometric and hyperbolic function solutions): When $\beta=0$,

$$
\begin{aligned}
& u_{13,14}= \pm \sqrt{-\frac{6 a^{2}+6 b^{2}+6 c^{2}}{\delta}} \sqrt{\alpha \gamma} \\
& \times \tan \left(\sqrt { \alpha \gamma } \left(\frac{a x^{\alpha}}{\Gamma(1+\alpha)}+\frac{b y^{\alpha}}{\Gamma(1+\alpha)}+\frac{c z^{\alpha}}{\Gamma(1+\alpha)}\right.\right. \\
& u_{15,16}= \pm \sqrt{-\frac{6 a^{2}+6 b^{2}+6 c^{2}}{\delta}} \sqrt{-\alpha \gamma} \\
& \times \tanh \left(\sqrt { - \alpha \gamma } \left(\frac{a x^{\alpha}}{\Gamma(1+\alpha)}+\frac{b y^{\alpha}}{\Gamma(1+\alpha)}+\frac{c z^{\alpha}}{\Gamma(1+\alpha)}\right.\right. \\
&\left.\left.-\left(a^{2} \gamma+b^{2} \gamma\right) \frac{2 \alpha a t^{\alpha}}{\Gamma(1+\alpha)}\right)\right), \\
&\left.\left.\left.g_{2}+c^{2} \gamma\right) \frac{2 \alpha a t^{\alpha}}{\Gamma(1+\alpha)}\right)\right) .
\end{aligned}
$$

Case 3 (Trigonometric function solution): When $\beta^{2}<4 \alpha \gamma$ and $\gamma \neq 0$,

$$
\begin{aligned}
u_{17,18}= & \pm \frac{1}{2} \sqrt{-\frac{\left(6 a^{2}+6 b^{2}+6 c^{2}\right)\left(4 \alpha \gamma-\beta^{2}\right)}{\delta}} \\
& \times \tan \left(\frac { 1 } { 2 } \sqrt { 4 \alpha \gamma - \beta ^ { 2 } } \left(\frac{a x^{\alpha}}{\Gamma(1+\alpha)}+\frac{b y^{\alpha}}{\Gamma(1+\alpha)}+\frac{c z^{\alpha}}{\Gamma(1+\alpha)}\right.\right. \\
& \left.\left.-\frac{1}{2}\left(a^{2}+b^{2}+c^{2}\right)\left(4 \gamma \alpha-\beta^{2}\right) \frac{a t^{\alpha}}{\Gamma(1+\alpha)}\right)\right) .
\end{aligned}
$$


Case 4 (Hyperbolic function solution): When $\beta^{2}>4 \alpha \gamma$ and $\gamma \neq 0$,

$$
\begin{aligned}
u_{19,20}= & \pm \frac{1}{2} \sqrt{-\frac{\left(6 a^{2}+6 b^{2}+6 c^{2}\right)\left(-4 \alpha \gamma-+\beta^{2}\right)}{\delta}} \\
& \times \tanh \left(\frac { 1 } { 2 } \sqrt { - 4 \alpha \gamma + \beta ^ { 2 } } \left(\frac{a x^{\alpha}}{\Gamma(1+\alpha)}+\frac{b y^{\alpha}}{\Gamma(1+\alpha)}+\frac{c z^{\alpha}}{\Gamma(1+\alpha)}\right.\right. \\
& \left.\left.-\frac{1}{2}\left(a^{2}+b^{2}+c^{2}\right)\left(4 \gamma \alpha-\beta^{2}\right) \frac{a t^{\alpha}}{\Gamma(1+\alpha)}\right)\right) .
\end{aligned}
$$

\section{Conclusion}

We have exact travelling wave solutions for the $(3+1)$-dimensional fractional modified KdV-Zakharov-Kuznetsov equation by the improved generalized tanh-coth method. All exact travelling wave solutions put back into the original equation by main of Maple software. This is confirm the validity of the solutions obtained in this paper.

\section{Acknowledgements}

The authors would like to the Department of Mathematics, Faculty of Science and Technology, Loei Rajabhat University and the Department of Mathematics, Faculty of Applied Science, King Mongkuts University of Technology, North Bangkok.

\section{References}

[1] F. S. Khodadad, F. Nazari, M. Eslami and H. Rezazadeh, Soliton solutions of the conformable fractional Zakharov-Kuznetsov equation with dual-power law nonlinearity, Optical and Quantum Electronics 49(11) (2017); Article 384, 12 pages.

DOI: https://doi.org/10.1007/s11082-017-1225-y

[2] M. Eslami, H. Rezazadeh, M. Rezazadeh and S. S. Mosavi, Exact solutions to the space-time fractional Schrödinger-Hirota equation and the space-time modified KdV-Zakharov-Kuznetsov equation, Optical and Quantum Electronics 49(8) (2017); Article 279, 15 pages.

DOI: https://doi.org/10.1007/s11082-017-1112-6 
[3] S. Sahoo and S. S. Ray, Analysis of Lie symmetries with conservation laws for the $(3+1)$ dimensional time-fractional mKdV-ZK equation in ion-acoustic waves, Nonlinear Dynamic 90(2) (2017), 1105-1113.

DOI: https://doi.org/10.1007/s11071-017-3712-x

[4] Y. Cenesiz, O. Tasbozan and A. Kurt, Functional variable method for conformable fractional modified KdV-ZK equation and Maccari system, Tbilisi Mathematical Journal 10(1) (2017), 117-125.

[5] S. Sahoo and S. S. Ray, Improved fractional sub-equation method for (3+1)-dimensional generalized fractional KdV-Zakharov-Kuznetsov equations, Computers and Mathematics with Applications 70(2) (2015), 158-166.

DOI: https://doi.org/10.1016/j.camwa.2015.05.002

[6] M. T. Islam, M. A. Akbar and M. A. K. Azad, Closed-form travelling wave solutions to the nonlinear space-time fractional coupled Burgers' equation, Arab Journal of Basic and Applied Sciences 26(1) (2019), 1-11.

DOI: https://doi.org/10.1080/25765299.2018.1523702

[7] W. Malfliet, Solitary wave solutions of nonlinear wave equation, American Journal of Physics 60(7) (1992), 650-654.

DOI: https://doi.org/10.1119/1.17120

[8] E. Fan and Y. C. Hona, Generalized tanh method extended to special types of nonlinear equations, Zeitshrift fur Naturforschung A 57(8) (2002), 692-700.

DOI: https://doi.org/10.1515/zna-2002-0809

[9] A. M. Wazwaz, The extended tanh method for new solitons solutions for many forms of the fifth-order KdV equations, Applied Mathematics and Computation 84(2) (2007), 1002-1014.

DOI: https://doi.org/10.1016/j.amc.2006.07.002

[10] C. A. Gomez and A. H. Salas, The Cole-Hopf transformation and improved tanh-coth method applied to new integrable system (KdV6), Applied Mathematics and Computation 204(2) (2008), 957-962.

DOI: https://doi.org/10.1016/j.amc.2008.08.006

[11] G. Garzon and S. Gomez, The generalized tanh-coth method applied to biological model referent to nano-solitons of ionic wave, Applied Mathematical Sciences 9(138) (2015), 6877-6882.

[12] M. Torvattanabun and S. Koonprasert, Variational iteration method combined with the improved generalized tanh-coth method for the generalized $(1+1)$-dimensional and $(2+1)$ dimensional equations, Far East Journal of Mathematical Sciences 100(8) (2016), 1339-1355.

DOI: http://dx.doi.org/10.17654/MS100081339 
[13] M. Torvattanabun, J. Simmapim, D. Saennuad and T. Somaumchan, The improved generalized tanh-coth method applied to sixth-order solitary wave equation, Journal of Mathematics (2017); Article ID 8751097, 11 pages.

DOI: https://doi.org/10.1155/2017/8751097

[14] X.-J. Yang, Advanced Local Fractional Calculus and its Applications, World Science Publisher, New York, 2012.

[15] X.-J. Yang, J. A. T. Machado and H. M. Srivastava, A new numerical technique for solving the local fractional diffusion equation: Two-dimensional extended differential transform approach, Applied Mathematics and Computation 274 (2016), 143-151.

DOI: https://doi.org/10.1016/j.amc.2015.10.072

[16] S. Bibi, S. T. M. Din, R. Ullah, N. Ahmed and U. Khan, Exact solutions for STO and $(3+1)$-dimensional KdV-ZK equations using $\left(\frac{G^{\prime}}{G^{2}}\right)$-expansion method, Results in Physics 7 (2017), 4434-4439.

DOI: https://doi.org/10.1016/j.rinp.2017.11.009 